\title{
CSF STREM2 AND TAU WORK TOGETHER IN PREDICTING INCREASED \\ TEMPORAL LOBE ATROPHY IN OLDER ADULTS
}

Running title: sTREM2 and brain atrophy in aging

Nathalie Bodd Halaas ${ }^{1,2,3}$, Kristi Henjum ${ }^{1,3,4}$, Kaj Blennow ${ }^{5}$, Shams Dakhil ${ }^{1,3}$, Ane-Victoria Idland $^{1,2,3}$, Lars N.G. Nilsson ${ }^{4}$, Donatas Sederevicius ${ }^{2,6}$, Didac Vidal-Piñeiro ${ }^{2}$, Kristine B Walhovd $^{2,6}$, Torgeir Brunn Wyller ${ }^{1,3}$, Henrik Zetterberg ${ }^{7,8,9,10}$, Leiv Otto Watne ${ }^{1}$, Anders M Fjell $^{2,6}$.

${ }^{1}$ Oslo Delirium Research Group, Department of Geriatric Medicine, Oslo University Hospital, Oslo, Norway

${ }^{2}$ Center for Lifespan Changes in Brain and Cognition, Department of Psychology, University of Oslo, Oslo, Norway

${ }^{3}$ Institute of Clinical Medicine, University of Oslo, Oslo, Norway

${ }^{4}$ Department of Pharmacology, Institute of Clinical Medicine, University of Oslo Oslo University Hospital, Oslo, Norway

${ }^{5}$ Department of Psychiatry and Neurochemistry, Sahlgrenska Academy, University of Gothenburg, Mölndal, Sweden

Clinical Neurochemistry Laboratory, Sahlgrenska University Hospital, Mölndal, Sweden

${ }^{6}$ Department of Radiology and Nuclear Medicine, Oslo University Hospital, Oslo, Norway

${ }^{7}$ Department of Psychiatry and Neurochemistry, Institute of Neuroscience and Physiology, the Sahlgrenska Academy at the University of Gothenburg, Mölndal, Sweden 
${ }^{8}$ Clinical Neurochemistry Laboratory, Sahlgrenska University Hospital, Mölndal,

Sweden

${ }^{9}$ Department of Neurodegenerative Disease, UCL Institute of Neurology, Queen Square, London, United Kingdom

${ }^{10}$ UK Dementia Research Institute at UCL, London, United Kingdom

\section{Corresponding author:}

Nathalie Bodd Halaas, University of Oslo, Campus Ullevål, Department of Geriatric Medicine

PB 4956 Nydalen

0424 Oslo, Norway

Telephone: +4798640857

E-mail: nathaliebodd@gmail.com 


\begin{abstract}
Neuroinflammation may be a key factor in brain atrophy in aging and age-related neurodegenerative disease. The objective of this study was to test the association between microglial expression of soluble Triggering Receptor Expressed on Myeloid Cells 2 (sTREM2), as a measure of neuroinflammation, and brain atrophy in cognitively unimpaired older adults. Brain MRIs and CSF sTREM2, total tau (t-tau), phosphorylated ${ }_{181}$ tau (p-tau) and $A \beta 42$ were analyzed in 115 cognitively unimpaired older adults, classified according to the A/T/(N)-framework. MRIs were repeated after two $(n=95)$ and four $(n=62)$ years. High baseline sTREM2 was associated with accelerated cortical thinning in the temporal cortex of the left hemisphere, as well as bilateral hippocampal atrophy, independently of age, A $\beta 42$ and tau. sTREM2-related atrophy only marginally increased with biomarker positivity across the $\mathrm{AD}$ continuum $(\mathrm{A}-\mathrm{T}-\rightarrow \mathrm{A}+\mathrm{T}-\rightarrow \mathrm{A}+\mathrm{T}+$ ) but was significantly stronger in participants with a high level of p-tau $(\mathrm{T}+)$. sTREM2-related cortical thinning correlated significantly with areas of high microglial-specific gene expression in the Allen Human Brain Atlas. In conclusion, increased CSF sTREM2 was associated with accelerated cortical and hippocampal atrophy in cognitively unimpaired older participants, particularly in individuals with tau pathology. This suggests a link between neuroinflammation, neurodegeneration and amyloid-independent tauopathy.
\end{abstract}

Key words: aging, Alzheimer's Disease, brain atrophy, neuroinflammation, tau. 


\section{INTRODUCTION}

Neuroinflammation is assumed to be an important factor in neurodegeneration in age-related conditions such as Alzheimer's Disease (AD) (McManus and Heneka 2017; Regen et al. 2017). However, structural brain changes are seen also in cognitively unimpaired older adults, and frontotemporal cortices and hippocampi are commonly affected by atrophy in ageing (Fjell et al. 2013). These changes do not appear to be totally benign but are rather related to reduced cognitive functions (Nyberg et al. 2012; Pudas et al. 2013; Fjell et al. 2014; Gorbach et al. 2017). The pathophysiological mechanisms of age-related neurodegeneration remain poorly understood. Microglial responses may exacerbate and contribute to brain atrophy in aging and the development of neurodegenerative disease (NDD) (Heneka et al. 2015; Ransohoff 2016; Wyss-Coray 2016; Jay et al. 2017; Hickman et al. 2018). Microglial activity may be measured in vivo by levels of soluble Triggering Receptor Expressed on Myeloid Cells 2 (sTREM2) in the CSF. TREM2 is a transmembrane receptor protein expressed predominantly by microglia in the CNS, and sTREM2 is a soluble fragment of TREM2 which is cleaved from the membrane-bound protein and enters the CSF (Kleinberger et al. 2014; Heslegrave et al. 2016; Jay et al. 2017). Loss-of-function mutations in TREM2 enhance the risk of NDDs, including AD (Guerreiro et al. 2013; Jay et al. 2017; Carmona et al. 2018). Effects of TREM2 expression on NDD are complex, probably depending on microglial activation state and pathology at different disease stages (Jay et al. 2017; Carmona et al. 2018).

If neuroinflammation is a factor of importance in age-related brain atrophy, we would expect sTREM2 levels to be associated with brain degeneration over time. Few studies have examined the association between sTREM2 and longitudinal structural cortical changes in cognitively unimpaired older adults classified along the AD continuum. A large-cross 
sectional study of participants across the AD spectrum found that sTREM2 was associated with tau-related neurodegeneration but not with amyloid- $\beta$ pathology (Suarez-Calvet et al. 2019). In a study directly testing the relationship between sTREM2 and brain volumes, elevated CSF sTREM2 was related to increased gray matter volume and reduced diffusivity in the temporoparietal cortices and precuneus in mild cognitive impairment, suggesting brain swelling in relation to neuroinflammation (Gispert et al. 2016). However, a longitudinal study found no association between high versus low baseline CSF sTREM2 and hippocampal atrophy across the AD continuum (Rauchmann et al. 2018).

The aim of the present study was to test whether sTREM2 levels were related to brain atrophy across the cerebral cortex and the hippocampus in cognitively unimpaired older adults. Atrophy was measured with repeated MRI over up to four years, and unbiased statistical analyses were performed point-wise across the entire cortical mantle. We hypothesized that participants with high levels of sTREM2 would show more atrophy over time. The resulting statistical maps, representing sTREM2-related atrophy, were tested against gene expression maps from the Allen Human Brain Atlas (Allen Institute for Brain Science; http://www.brainmap.org (Hawrylycz et al. 2012)). We hypothesized that there would be a spatial correlation between sTREM2-related cortical thinning and microglial-specific gene expression. We also characterized participants according to the research classification of AD published by the National Institute on Ageing and Alzheimer' Association (NIA-AA), the A/T/(N)-framework, focusing on biomarkers of amyloid/tau aggregation and neurodegeneration (Jack et al. 2018). This allowed us directly to test whether a relationship between sTREM2 and brain atrophy would interact with established AD biomarker profiles.

\section{MATERIALS AND METHODS}




\section{Sample}

The study was conducted in accordance with the Declaration of Helsinki and approved by the Regional Committee for Ethics in Medical Research in Norway (REK, 2011/2052). All participants provided written informed consent. 172 patients undergoing elective gynecological (genital prolapse), urological (benign prostate hyperplasia, prostate cancer, or bladder tumor/cancer) and orthopedic (knee or hip replacement) surgery in spinal anesthesia, aged 65 years or older the year of inclusion, were recruited to the COGNORM-study from 2012-2013 at Oslo University Hospital and Diakonhjemmet Hospital, Oslo, as previously described (Idland et al. 2016). Dementia, previous stroke with sequelae, Parkinson's disease and other acknowledged or suspected brain disease likely to influence cognition were exclusion criteria at baseline. Participants were evaluated with a comprehensive battery of cognitive tests prior to surgery, comprising the Mini Mental Status Examination (MMSE) (Folstein et al. 1975), Clock Drawing Test (Aprahamian et al. 2009), Word List Memory Task (Morris et al. 1989), Trail Making Test A and B (Reitan 1955), Kendrick Object Learning Test (Kendrick et al. 1979) and verbal fluency (The Controlled Word Association Test, with the letters F, A and S, and Animal Naming) (Spreen 1991), yielding 11 test scores. Cognitive testing was repeated yearly, with a comparable test battery. To select cognitively unimpaired participants at baseline, we excluded patients with 1) an MMSE score $<28$ and more than one other test with a score more than 1.5 SD below the mean normal value for age, sex, and educational level $(n=3)$ and 2) suspected undiagnosed dementia with referral to a memory clinic (based on test scores and clinical data, $\mathrm{n}=6$ ).

CSF at baseline was collected in 155 participants at the onset of anesthesia prior to administration of the anesthetic agent. 14 participants underwent a second lumbar puncture with CSF sampling after a mean of 4.5 years. MRI was performed at baseline after surgery in 
128 participants, with a mean time between CSF collection and MRI acquisition of 60 days. MRI was repeated twice with a mean follow up time of $2.1(1.7-2.8, \mathrm{SD}=0.22)$ and $4.3(3.9$ $4.7, \mathrm{SD}=0.23)$ years. The final sample consisted of 115 cognitively unimpaired participants with sTREM2 and MRIs at baseline, of whom 95 had two MRIs and 62 had three MRIs. The 20 participants with MRI only at baseline could still be included in the statistical analyses due to the use of a mixed model approach (see below). Of these, two participants lacked tau and A $\beta 42$ CSF measures.

\section{APOE genotyping}

Blood samples were genotyped for APOE (gene map locus 19q13.2) using TaqMan Allelic Discrimination technology (Applied Biosystems, Carlsbad, CA, USA). Genotypes were obtained for the two SNPs that are used to unambiguously define the $\varepsilon 2, \varepsilon 3$, and $\varepsilon 4$ alleles (rs7412 and rs429358).

\section{CSF Sampling and biochemical analyses}

CSF was collected in polypropylene tubes, centrifuged, aliquoted and stored at $-80^{\circ} \mathrm{C}$, as described elsewhere (Idland et al. 2016). Samples for sTREM2 and AD biomarker analysis were sent on dry ice to the laboratories without information about clinical data (masked data). CSF AD biomarkers, CSF A $\beta 42$, phosphorylated ${ }_{181}$ tau (p-tau) and total tau (t-tau), were determined using INNOTEST enzyme-linked immunosorbent assays (ELISA; Fujirebio, Ghent, Belgium) at Sahlgrenska University Hospital (Mölndal, Sweden) by board-certified laboratory technicians, as previously described (Idland et al. 2016). CSF sTREM2 was assayed by an in-house sandwich ELISA at Oslo University Hospital, as described in a previous study (Henjum et al. 2016). To avoid any inter-assay variation afflicting the results 
of samples with longitudinal measures, CSF sTREM2 levels were determined simultaneously within the same assay.

\section{Biomarker profiling: The AT-framework}

Participants were classified according to the A/T/(N)-framework (Jack, Bennett, et al. 2016) by applying CSF measures with the following cut-off values: $\mathrm{A}+<\mathrm{A} \beta 42530 \mathrm{pg} / \mathrm{ml}<\mathrm{A}-$ and $\mathrm{T}+>$ p-tau $60 \mathrm{pg} / \mathrm{ml}>\mathrm{T}$ - as established for the laboratory (Hansson et al. 2006). 30 participants were $\mathrm{A}+$ and $83 \mathrm{~A}-, 48 \mathrm{~T}+$ and $65 \mathrm{~T}-$. Along the proposed $\mathrm{AD}$ spectrum, 50 had the A-T- biomarker profile ("normal AD biomarkers"), 15 had A+T- (“AD pathological change") and 15 had A+T+ ("AD”). In addition, 33 corresponded to the A-T+ profile ("NonAD pathological change").

\section{MRI acquisition and processing}

T1-weighted MPRAGE 3D images were acquired with a 1.5 T Siemens Avanto scanner using a 12-channel head coil $(\mathrm{TR}=2400 \mathrm{~ms}, \mathrm{TE}=3.79 \mathrm{~ms}$, Field of View $=240 \mathrm{~mm}$, slice thickness $=1.20 \mathrm{~mm}$, pixel size $=1.25 \times 1.25 \mathrm{~mm}$ ). Images were processed with the longitudinal stream in FreeSurfer 6.0 (FS) (https://surfer.nmr.mgh.harvard.edu), described elsewhere (Dale et al. 1999; Fischl et al. 2002; Reuter et al. 2012; Jovicich et al. 2013), generating maps of cortical thickness and hippocampal volume. Since FS is an almost fully automated processing tool, manual editing was not performed to avoid introducing errors, except checking for gross registration and segmentation-errors. Maps were smoothed using a circularly symmetric Gaussian kernel with a full width at half maximum of $15 \mathrm{~mm}$ (Fischl et al. 1999) before being entered into statistical analyses. 
We used a "virtual histology" approach, as described below, to test how anatomical differences in sTREM2-related cortical thinning related to inter-regional gene expression profiles associated with specific cell types, estimated ex-vivo. The analysis provides information on the specific types of cells possibly involved in sTREM2-related thinning and thus facilitates a neurobiological interpretation.

As reported in detail elsewhere (French and Paus 2015; Shin et al. 2018), gene-expression data were obtained in ex-vivo brains from the Allen Human Brain Atlas (Allen Institute for Brain Science; http://www.brain-map.org(Hawrylycz et al. 2012)), and summarized into the 34 cortical regions of the Desikan/Killiany Atlas (Desikan et al. 2006) by use of MNI152 coordinates. Subsequently, a two-step procedure was applied to remove genes with inconsistent regional expression profiles. Firstly, for each gene, the consistency of the interregional expression profile was evaluated with the mean Spearman correlation between each of the donor's profiles and the median profile of that gene (French and Paus 2015). Secondly, the mean profiles of gene expression between the Allen and the BrainSpan atlases (www.brainspan.org) were compared across the 11 homologous cortical regions(Shin et al. 2018). From the 20737 genes profiled in the Allen Human Brain Atlas, 2511 genes were retained as showing consistent gene-expression regional profiles. A list of genes expressed in specific cell types was obtained from Zeisel et al ${ }^{33}$. The list of cell-specific genes was intersected with the panel of genes with consistent profiles $(n=2511)$ after which the following number of genes per cell-type remained: S1 pyramidal neurons ( $\mathrm{n}=73), \mathrm{CA} 1$ pyramidal neurons $(n=103)$, interneurons $(n=100)$, astrocytes $(n=54)$, microglia $(n=48)$, oligodendrocytes $(n=60)$, ependymal $(n=84)$, endothelial $(n=57)$, and mural $(n=25)$.

\section{Statistical analyses}


Spatiotemporal linear mixed effects models were run to test the relationship between cortical thickness change and baseline levels of sTREM2 by a matlab add-on to FreeSurfer (BernalRusiel, Greve, et al. 2013; Bernal-Rusiel, Reuter, et al. 2013). Thickness at each vertex and time point was used as dependent variable, with random intercept, and time from baseline, sTREM 2 and the time $\times$ sTREM 2 interaction as predictors of interest, with age and sex as nuisance covariates in all analyses. Significance of the time $\times$ sTREM2 interaction term was taken as evidence for an effect of sTREM2 on cortical thickness over time, i.e. cortical thinning or atrophy. For volumetric analyses (hippocampus), intracranial volume was used as an additional covariate. All continuous predictor variables were z-transformed before being entered into the models. Separate models were run with A $\beta 42, \mathrm{~A} \beta 42 \times$ sTREM2, total-tau, total-tau $\times$ sTREM2, and A $\beta 42$ and total-tau, as additional covariates. Surface results were tested against an empirical null distribution of maximum cluster size across 10000 iterations using Z Monte Carlo simulations, synthesized with a cluster-forming threshold of $\mathrm{p}<0.01$ (two-sided), yielding results corrected for multiple comparisons across space. Thickness values for each participant and time point from the clusters surviving statistical correction were extracted and used for post-hoc analyses.

Post hoc analyses included generalized additive mixed models (GAMM) with the same variables as above, to visualize change trajectories and test interactions, run in $\mathrm{R}$ (https://www.r-project.org) using Rstudio IDE with the package "mgcv" (www.rstudio.com) (Wood 2006). GAMMs were also run to test relationship between sTREM2 and hippocampal volume and memory performance over time. Separate GAMMs were run including p-tau, ptau $\times$ time and sTREM $2 \times p$-tau $\times$ time to explore the relationship between sTREM 2 and tau in predicting atrophy. We also tested whether presence of the APOE $\varepsilon$-allele affected the sTREM2-atrophy relationships by adding APOE status as an additional covariate in the main 
analyses. To test whether the participants' biomarker profile affected sTREM2-related atrophy, GAMMs with sTREM2×time by biomarker-profile as an additional 3-way tensor interaction were run, including also all main effects and lower order interactions in the same models.

To test the association between sTREM2-related thinning and the cell-type expression profiles, the vertex-wise parameter estimates (Beta-coefficients) of the left cortical surface from the sTREM2 - cortical thinning analysis were summarized into the 34 regions of interest and multiplied by -1 so that positive values corresponded to more cortical thinning with higher sTREM2. A Pearson's correlation was performed between the Beta-coefficients and the profile of gene expression for each marker gene across the regions. We used the average expression-thinning correlation for each panel of genes (each cell-type) (Shin et al. 2018). For each cell-type panel, we obtained the empirical null distribution of the test statistic by iteratively ( $\mathrm{n}=10.000)$ selecting a random number corresponding to the number of genes included in the cell-type panel, calculating their expression-thinning correlation coefficients and mean average value. Based on the empirical null distribution, we obtained two-sided pvalues for each cell-type panel, which was further False Discovery Rate adjusted for multiple testing ( $n=9$ cell-type panels).

As many participants were diagnosed with conditions commonly treated with antiinflammatory drugs, we assessed whether use of antiinflammatory drugs at baseline was related to participants' levels of sTREM2 in the CSF in post hoc analyses. We defined antiinflammatory drugs as those classified as MO1A (antiinflammatory and antirheumatic products, non-steroids), MO1B (antiinflammatory and antirheumatic products) or H02 
(corticosteroids for systemic use) according to the Anatomical Therapeutic Chemical (ATC) Classification System (https://www.whocc.no/atc_ddd_index/).

\section{RESULTS}

\section{Population demographics}

Age correlated positively with increased sTREM2 levels both in cross-sectional and longitudinal analysis (mean annual increase of sTREM2 of approximately $10 \%$ ). Population demographics are described in Table 1.

\section{Relationship between sTREM2 and brain atrophy}

Higher levels of sTREM2 were related to accelerated cortical thinning in three clusters located in the lateral and inferior left hemisphere temporal cortex, covering 1578, 830 and 753 $\mathrm{mm}^{2}$ (Cluster-wise $\mathrm{p}$-value cluster $1=0.0001$, cluster $2=0.020$ and cluster $3=0.011$, Figure 1A). Effects survived statistical corrections in the left hemisphere only. To assess whether the association between sTREM2 and cortical thinning was unilateral or whether the observed asymmetry was an effect of the statistical threshold, we inspected the uncorrected statistical maps thresholded at $\mathrm{p}<0.05$ (see Supplemental Information). These revealed that although effects were clearly more extended in the left hemisphere, higher levels of sTREM2 were also related to more thinning in the right hemisphere, although these effects did not survive statistical corrections. Thus, the results should not be interpreted as supporting a unilateral effect of sTREM2 on cortical thinning.

Post hoc GAMMs were run for each cluster and the time $\times$ sTREM2 interactions visualized by contour plots (Figure $2 \mathrm{~A}$ ). These revealed that the time $\times$ sTREM2 interaction on cortical thinning was driven by participants with high sTREM2 levels. Thus, for illustrative purposes we dichotomized the sample by $Z \geq 1$ (32 observations) vs. $Z<1$ (240 observations), and 
estimated change-slopes in each group (see Figure 2B-C). As can be seen, high sTREM2 participants showed highly significant close to linear thinning in all 3 clusters ( $F$ for cluster 1 $=18.5,2=25.8$ and $3=16.9$; all p's $<0.0005$ ). In contrast, despite higher power, participants with normal sTREM2 level showed marginally significant thinning in cluster 1 only $(F=4.12$, $\mathrm{p}=0.04)$

Additional GAMMs were run testing the effect of sTREM2 on hippocampal atrophy. For both left $(\mathrm{F}=3.97, \mathrm{p}<0.05)$ and right $(\mathrm{F}=3.14, \mathrm{p}<0.02)$ hippocampus, higher baseline levels of sTREM2 were related to higher atrophy rates (Figure 3). We performed post hoc tests dividing participants in groups of "high" vs. "normal" sTREM2, based on either a mean split or above/ below $1 \mathrm{SD}$ over the mean. The group analyses revealed no significant effect of sTREM2 on hippocampus volume change (all p's $>0.34$ ), demonstrating that dichotomizing sTREM2 yields reduced sensitivity, and that sTREM2 is best treated as a continuous variable in predicting hippocampal atrophy.

To test whether presence of the APOE $\varepsilon 4$-allele affected the relationships, all GAMMS were re-run with APOE as an additional covariate. This did not affect the sTREM2-atrophy relationships, and APOE was not significant in predicting atrophy for any of the tested regions.

\section{Effects of tau and AB42 on sTREM2-related atrophy}

To assess whether the sTREM2-related cortical thinning was dependent on the biomarkers $\mathrm{A} \beta 42$, $\mathrm{t}$-tau or $\mathrm{p}$-tau, the spatiotemporal linear mixed effects models were re-run including $\mathrm{A} \beta 42 \mathrm{and} /$ or tau as additional covariates. sTREM2 correlated positively with $p$-tau $(\rho=0.51$, $\mathrm{p}<0.001)$ and t-tau $(\rho=0.52, \mathrm{p}<0.001)$, but not with $\mathrm{A} \beta 42(\rho=0.11, \mathrm{p}=0.25)$. T-tau and $\mathrm{p}$-tau were almost completely collinear $(\rho=0.97, p<0.001)$, and could therefore be considered equivalent in subsequent statistical analyses. Since t-tau showed a numerically slightly higher 
correlation with sTREM2 than did p-tau, t-tau was included as covariate in this model to ensure the most conservative test of the relationship between sTREM2 and cortical thinning. The effects of sTREM2 on cortical thinning were not reduced by regressing out A $\beta 42$ levels (see Figure 1B). The total spatial extension of sTREM2 effects with A $\beta 42$ regressed out was $3405 \mathrm{~mm}^{2}$, compared to $3161 \mathrm{~mm}^{2}$ in the original model where A $\beta 42$ was not included. The A $\beta 42 \times$ sTREM2 interaction term was not significantly related to cortical thinning. The analyses were repeated with both $\mathrm{A} \beta 42$ and t-tau as covariates (Figure 1C). This influenced the spatial extension of the effects, reducing the significant effect area with $41.3 \%$ compared to the initial model.

Since tau accounted for a substantial part of the sTREM2-related cortical atrophy, we ran additional analyses to disentangle the effects of both biomarkers. For the three cortical regions and the hippocampus, including p-tau as an additional covariate still yielded highly significant effects of sTREM2 on cortical thinning (cluster $1: \mathrm{F}=25.44, \mathrm{p}=8.37 \mathrm{e}^{-7}$; cluster $2: \mathrm{F}=19.32$, $\mathrm{p}=1.59 \mathrm{e}^{-5} ;$ cluster $\left.3: \mathrm{F}=16.99, \mathrm{p}=5.02 \mathrm{e}^{-5}\right)$. Thus, we ran additional GAMMs directly testing the relative contributions of sTREM2 and p-tau on atrophy, and also exploring interactions between sTREM2 and p-tau. For none of the clusters was p-tau related to atrophy when included in the same model as sTREM2 (all p's > .1), while sTREM2 still was (all p's $<.05)$. Interestingly, for clusters 2 , there was a significant interaction between sTREM2 and $\mathrm{p}$-tau $(\mathrm{F} 2.31, \mathrm{p}<.05)$. Follow-up analyses showed that $\mathrm{p}$-tau was related to more atrophy in the high-sTREM2 group $(\mathrm{F}=3.89, \mathrm{P}=.022)$ but not in the low sTREM2-group $(\mathrm{F}=0.05, \mathrm{p}$ $=.83)$.

The same analyses were run for hippocampal atrophy. Adding p-tau as a covariate, sTREM2 was still significantly related to hippocampal atrophy (left hippocampus: $F=3.97, p<05$ : 
right hippocampus: $\mathrm{F}=3.32, \mathrm{p}<.02$ ). P-tau was not significantly related to rate of change (left hippocampus: $\mathrm{F}=0.02, \mathrm{p}=.89$; right hippocampus: $\mathrm{F}=0.13, \mathrm{p}=.72$ ). For both hemispheres, we found a significant interaction between sTREM2 and p-tau in prediction of atrophy (left: $\mathrm{F}=3.83, \mathrm{p}<.02$; right: $\mathrm{F}=7.32, \mathrm{p}=8.47 \mathrm{e}^{-5}$ ). This interaction was caused by $\mathrm{p}$ tau being more predictive of atrophy in the high sTREM2 group (right: $F=4.79, p=.039$; left: $F=3.32, p=.052$ ) than the low sTREM2 group (right: $F=1.26, p=.26 ;$ left: $F=0.61, p$ $=.44)$.

\section{Longitudinal sTREM2 analyses}

Longitudinal sTREM2 data were available for a smaller subset of participants $(n=14$, mean interval 4.27 years, $\mathrm{SD}=0.24$ ). Due to the very low sample size, these results must be treated with great caution, but are included since there is very little existing knowledge about how sTREM2 changes over time. sTREM2 increased significantly (Baseline: 7.54, $\mathrm{SD}=3.87$; Follow-up $10.64, \mathrm{SD}=5.98, \mathrm{t}=4.04, \mathrm{p}=0.001)$ and correlated strongly between time points $\left(\mathrm{r}=0.92, \mathrm{p}=0.00 \mathrm{E}^{-06}\right)$. Notably all samples displayed an increase. Change in sTREM2 also correlated with both baseline $(\mathrm{r}=0.56, \mathrm{p}=0.035)$ and follow-up $(\mathrm{r}=0.85, \mathrm{p}=0.0001)$ values.

Acknowledging the small sample, tentative GAMMS were run, testing the relationship between sTREM2 changes (Follow-up - Baseline values) and hippocampal atrophy. For both left and right hippocampus, a significant sTREM2 change $\times$ time interaction was found (Left: $\mathrm{F}=21.61, \mathrm{p}=4.64 \mathrm{e}^{-05}$; Right: $\mathrm{F}=11.72, \mathrm{p}=0.002$ ), showing more atrophy in participants with more increase in STREM2. This fits with the baseline results, but must still be regarded as tentative due to the small sample size. Similar analyses were run for the three cortical clusters identified in Figure 1. We did not see any significant relationships between thinning and sTREM2 increases (all p's $>0.10$ ), likely due to low statistical power. 


\section{Biomarker profiling}

GAMMs were run to test whether the sTREM2-associated cortical thinning and hippocampal atrophy differed as a function of AT biomarker profile, comparing A-/T- vs. A+/T- vs. $\mathrm{A}+/ \mathrm{T}+$. The only significant difference was found for left hippocampus, where sTREM2 was more strongly related to atrophy in the $\mathrm{A}+/ \mathrm{T}+$ group than the $\mathrm{A}-/ \mathrm{T}-$ group $(\mathrm{F}=6.83, \mathrm{p}=$ 0.01). Further, we compared A-/T+ (suspected non-Alzheimer pathology; SNAP) to A+/T(Alzheimer's continuum). For all regions, sTREM2 was more strongly related to atrophy in the A-/T+ group, with the differences being significant for Cluster $2(\mathrm{~F}=7.90, \mathrm{p}=0.006)$ and right hippocampus $(\mathrm{F}=8.67, \mathrm{p}=0.002)$.

\section{Relationship between sTREM2 and memory change}

GAMMs were run with memory score as dependent variable, with 515 observations spread over 5 time-points, age as predictor, controlling for sex, with time point as an additional covariate to control for retest effects (Figure 4). Age had a linear negative effect on memory $(\mathrm{t}=-4.7, \mathrm{p}=3.46 \mathrm{e}-06)$. Adding baseline sTREM2 as an additional predictor, there was no significant main effect of sTREM2 levels on memory score $(F=1.91, p=0.17)$, nor a significant effect of sTREM2 on memory change $(\mathrm{F}=1.59, \mathrm{p}=0.21)$.

\section{Relationship between sTREM2-related thinning and cell-specific gene-expression profiles}

The sTREM2-related cortical thinning profile was correlated with the expression profiles of cell-specific genes. The results are shown in Figure 5 and Table 2. The average correlation for the CA1-pyramidal neurons $(\mathrm{p}<0.001)$ and microglia $(\mathrm{p}=0.03)$ cell-types significantly differed from the empirical null distributions (False Discovery rate-corrected $<0.05$ ). Both distributions were shifted towards positive coefficients, i.e. the cortical regions associated 
with steeper sTREM2-related cortical thinning showed higher expression of CA1-pyramidal and microglia-specific genes.

\section{Antiinflammatory drugs and sTREM2 levels}

No difference in CSF sTREM2 levels was found between participants not medicated with $(n=80$, median $(I Q R) 8.4(6.2-10.6))$ and medicated with antiinflammatory drugs $(n=35$, median (IQR) 9.5 (6.4-10.8)), Mann Whitney, $\mathrm{p}=0.32$ (U=1582). No difference in sTREM2 levels was found between participants medicated only with non-steroid antiinflammatory and antirheumatic drugs (M01A, $\mathrm{n}=25$, median 9.5), only systemic steroids (H02, $\mathrm{n}=7$, median= 7.3) or both (M01 and H02, $\mathrm{n}=3$, median=12.3), $\mathrm{p}=0.056$, in post hoc analysis. No participants were medicated with drugs of the MO1B-class.

\section{DISCUSSION}

In this study we evaluated the association between CSF sTREM2 and longitudinal changes on structural MRI in cognitively normal older adults. We showed that 1) a high baseline level of STREM2 was associated with accelerated cortical thinning in the lateral and inferior temporal cortex in the left hemisphere in areas with high microglial expression, 2) high baseline sTREM2 and greater longitudinal increases of sTREM2 were both associated with greater loss of hippocampal volume over time, 3 ) the association between sTREM2 and atrophy was independent of $A \beta$ and tau, but $p$-tau and sTREM2 interacted so that the effects of sTREM2 on atrophy was significantly stronger in participants with high levels of $\mathrm{p}$-tau and vice versa, and 4) baseline sTREM2 was not related to cognitive decline, suggesting that any clinical detrimental effect of sTREM2-associated atrophy was not detectable by our longitudinal memory testing. 
High levels of sTREM2 were associated with accelerated cortical thinning and greater loss of hippocampal volume over time. This relationship was independent of levels of t-tau, $p$-tau, $A \beta$ and APOE status, although the spatial extent of the sTREM2-related cortical atrophy was substantially reduced when controlling for levels of tau. Contrariwise, a recent study on participants along the $\mathrm{AD}$-continuum, dichotomizing participants based on high or low sTREM2 levels, revealed no association between sTREM2 and longitudinal hippocampal volume loss over a four-year period (Rauchmann et al. 2018). Our analyses indicate that sensitivity to detect relationships with atrophy may be lower when STREM2 is defined as a categorical variable, which may explain the divergent findings.

Microglial activation has traditionally been perceived as a secondary process to tissue damage/loss in healthy brains, firstly as a housekeeping function (Ransohoff 2016; Hickman et al. 2018). However, the activities of microglia and particularly the TREM2 receptor are more complex than previously thought (Jay et al. 2017; Carmona et al. 2018). A study in aged mice showed that phagocytosis of apoptotic neurons could induce subtypes of microglia with a reduced ability for homeostatic control and increased expression of neurodegenerationrelated genes through APOE-TREM2-dependent pathways (Krasemann et al. 2017). Also, a recent study on tauopathy in mice reported that TREM2-deficiency led to less microglial activation and atrophy in the temporal and piriform cortices, without affecting tau deposition/levels (Leyns et al. 2017). This suggests that an initially beneficial activation of microglia may become detrimental following a phenotypic shift in aged or NDD-related microglia, leading to a TREM2-mediated exacerbation of atrophy directly or through secondary noxious effects. Microglial TREM2 expression differs between brain regions in older individuals and the areas where we found most sTREM2-associated cortical thinning were found to correspond with known areas of high microglial expression through virtual 
histology (Forabosco et al. 2013). These areas are among the regions known to undergo accelerated thinning in cognitively normal older adults (Fjell et al. 2013). Elevated CSF sTREM2 has been linked to findings suggestive of brain edema in mild cognitive impairment in temporal areas (Gispert et al. 2016), indicating that sTREM2 may be involved in neuroinflammatory regulation in affected areas in early neurodegeneration. Moreover, high baseline levels of sTREM2 were associated with higher increases of sTREM2 over time, hypothetically indicating a phenotypical shift in microglial TREM2-expression. Taken together these findings strengthen the plausibility of sTREM2-mediated atrophy. In likelihood, neurodegeneration and neuroinflammation have reciprocal effects, each having the capability to bring about the other at different stages of neurodegeneration, potentially perpetuating a vicious cycle of deterioration.

\section{sTREM2-related atrophy is associated with high levels of p-Tau}

Pathological changes in AD are known to arise decades before symptomatic onset of the disease (Jack et al. 2010), with increased CSF t-tau and p-tau reflecting neurodegeneration and neuroaxonal tangle formation (Zetterberg 2017). Although the cascade of events leading to AD-pathological changes is still discussed, one hypothetical sequence of events promotes that amyloidosis, tangle formation and neurodegeneration occurs prior to microglial activation (Heneka et al. 2015). A biomarker-based research framework of AD has recently been proposed, featuring the $\mathrm{A} / \mathrm{T} / \mathrm{N}$-classification (Jack et al. 2018). Categorizing participants in A/T-groups, we found that biomarker positivity along the AD-continuum (A-T- $\rightarrow \mathrm{A}+\mathrm{T}-\rightarrow$ $\mathrm{A}+\mathrm{T}+$ ) increased sTREM2-related atrophy only in the $\mathrm{A}+/ \mathrm{T}+$ group, and only for the left hippocampus. Specifically, there appeared to be an APOE and amyloid-independent association between microglial sTREM2 -expression and cortical thinning. A $\beta$-independent sTREM2 expression has been described in other studies, supporting these findings (Suarez- 
Calvet, Kleinberger, et al. 2016; Rauchmann et al. 2018). While the association between sTREM2 and cortical thinning remained significant after correction for CSF tau, the spatial extent of the clusters was reduced by $41 \%$ after correction for tau concentrations, denoting some shared variance between tau, cortical thinning and sTREM2. Still, post hoc analyses revealed significant relationships between STREM2 and cortical and hippocampal atrophy also when controlling for levels of $\mathrm{p}$-tau. CSF t-tau has been shown to correlate with hippocampal atrophy and grey matter degeneration on structural MRI (Glodzik et al. 2012). A positive correlation between CSF t-/p-tau and sTREM2 levels has previously been demonstrated in cognitively normal older adults and in early stages of AD (Henjum et al. 2016; Suarez-Calvet et al. 2019).

Considering that the association between sTREM2 and atrophy partly depended on concentrations of CSF tau, and the fact that participants with high sTREM2-values ( $>1 \mathrm{SD}$ over mean) had significantly higher concentrations of both t-tau and p-tau than participants with low sTREM2, one might postulate that prior neuronal injury associated with AD-related tangle formations in the brain may have initiated increased sTREM2 expression. However, the interaction between sTREM2 and p-tau went both ways, and p-tau predicted atrophy in the tested regions only in participants with high levels of sTREM2. Also, it is important to note that although the relationship between sTREM2 and atrophy was spatially less extensive when tau levels were controlled for, robust significant relationships were still seen independently of tau. At large, research examining the pathophysiological relationship between sTREM2 and tau phosphorylation is scarce and the role of sTREM2 in tauopathy is still unsettled. A study evaluating the sequence of pathological changes in dominantly inherited $\mathrm{AD}$, demonstrated that increases in CSF t-tau and p-tau were closely associated with, but prior to, increases in CSF sTREM2, supporting that tauopathy may stimulate 
microglial sTREM2 expression (Suarez-Calvet, Araque Caballero, et al. 2016). On the other hand, a study visualizing microglia in relation to neurofibrillary structures in healthy and neurodegenerative human brain tissue supported that senescent (dystrophic) microglia were associated with and probably preceded tau pathology (Streit et al. 2009). Studies of tauopathy in mice are inconclusive, as some studies suggest a detrimental effect of TREM2-deficiency (Jiang et al. 2015; Bemiller et al. 2017), while others suggest that TREM2-deficiency may be protective (Jay et al. 2015; Leyns et al. 2017) of neurodegenerative changes and/or taupathology. The inconsistent findings suggest that (s)TREM2 may have a shifting role when NDDs progress, and tauopathy and microglial activation may have reciprocal effects. Our data establishes an association between neurodegeneration, tauopathy and sTREM2 expression in microglia, but do not permit us to determine causality. Increased knowledge concerning molecular mechanisms connecting atrophy and sTREM2 -associated microglial activity in humans is needed to further interpret causal relations.

\section{Primary age-related tauopathy and Suspected Non-Alzheimer disease Pathophysiology}

Primary age-related tauopathy (PART) refers to neurofibrillary tangles that are highly prevalent in autopsies of older brains in the absence of $A \beta$-accumulation and separated from AD. PART typically affects the medial temporal lobe and habitually progresses no further than the limbic Braak-stages (III-IV, including hippocampal affection) (Crary et al. 2014). Due to overlapping anatomical distribution of neurodegenerative changes, especially in the medial temporal lobe, PART is suspected to be one of the etiologies underlying a positive biomarker profile for tauopathy with normal $A \beta-$ levels $(\mathrm{A}-\mathrm{N}+)$, also called Suspected NonAlzheimer disease Pathophysiology (SNAP). (Jack, Knopman, et al. 2016). In cognitively normal older individuals, the proportion of SNAP increases with age and is consistently found to reach $1 / 4$ in the oldest old. In the study population, $29 \%$ of individuals had SNAP-profiles 
(median age 71) (table 1). PART may be associated with cognitive impairment and individuals with SNAP have an increased risk of progression to cognitive decline compared to biomarker-negative individuals. Nevertheless, both PART and SNAP appear to have less impact on cognition than AD (Crary et al. 2014; Jack, Knopman, et al. 2016). In this study the observed atrophy affected the temporal lobe (albeit not medially) and hippocampi and was related to tauopathy independently of abnormal amyloid levels. Furthermore, sTREM2-related atrophy was not associated with cognitive decline. However, the limited time of observation in conjunction with relatively high cognitive function at baseline, may have prevented subtle changes in cognitive function in relation to sTREM2 levels to be detected. With even longer follow up intervals or samples with lower cognitive baseline function, relationships between sTREM2 and cognitive decline might be observed. In sum, we postulate that the observed sTREM2-related atrophy may reflect PART as measured by SNAP. However, without visualization of tau-pathology, the association between SNAP and PART remains uncertain. Utilizing Tau-PET could test the hypothesis further and help elucidate a possible relationship between sTREM2, atrophy and tau-pathology.

\section{Limitations}

One weakness of our study is the lack of a good measure for participants' cerebrovascular disease load, as cerebrovascular disease may have shared variance with the degrees of atrophy and immunostimulation. Furthermore, although use of anti-inflammatory drugs was not found to influence the results, a possible inference of anti-inflammatory drugs on TREM2expression cannot be ruled out. This needs to be further investigated in future research. We have a relatively small study population at baseline, but substantial longitudinal data over several years strengthens our ability to detect change. Finally, longitudinal information on sTREM2 change was available only for a very small subsample, and these results must 
therefore be regarded as tentative and interpreted with great caution. Evaluation of cognitive changes was likely influenced by the learning effect

\section{Conclusion}

We found that high levels of microglial sTREM2 expression predicted accelerated cortical thinning and hippocampal volume loss. The association was APOE and amyloid-independent and partly related to tau phosphorylation, suggesting a link between PART or age-associatedatrophy and neuroinflammation.

\section{ACKNOWLEDGMENTS}

N.B. Halaas, K. Henjum, S. Dakhil, A-V. Idland, L.N.G. Nilsson, D. Sederevicius, D. VidalPiñeiro, K. Walhovd, T.B. Wyller, L.O. Watne and A.M. Fjell report no conflict of interests. K. Blennow has served as a consultant or at advisory boards for Alector, Alzheon, CogRx, Biogen, Lilly, Novartis and Roche Diagnostics, and is a co-founder of Brain Biomarker Solutions in Gothenburg AB, a GU Venture-based platform company at the University of Gothenburg, all unrelated to the work presented in this paper.

H. Zetterberg has served at scientific advisory boards for Roche Diagnostics, Wave, Samumed and $\operatorname{CogRx}$, has given lectures sponsored by Alzecure and is a co-founder of Brain Biomarker Solutions in Gothenburg AB, a GU Ventures-based platform company at the University of Gothenburg (all unrelated to the submitted work).

This work was supported by the Norwegian Health Association, the South-Eastern Norway Regional Health Authorities, the Medical Student Research Program of Norway and the Olav Thon Foundation. 
Corresponding author: Nathalie Bodd Halaas, University of Oslo, Campus Ullevål,

Department of Geriatric Medicine, PB 4956 Nydalen, 0424 Oslo, Norway; e-mail: nathaliebodd@gmail.com

\section{REFERENCES}

Aprahamian I, Martinelli JE, Neri AL, Yassuda MS. 2009. The Clock Drawing Test: A review of its accuracy in screening for dementia. Dementia \& neuropsychologia. 3:74-81.

Bemiller SM, McCray TJ, Allan K, Formica SV, Xu G, Wilson G, Kokiko-Cochran ON, Crish SD, LasagnaReeves CA, Ransohoff RM, Landreth GE, Lamb BT. 2017. TREM2 deficiency exacerbates tau pathology through dysregulated kinase signaling in a mouse model of tauopathy. Molecular neurodegeneration. $12: 74$

Bernal-Rusiel JL, Greve DN, Reuter M, Fischl B, Sabuncu MR, Alzheimer's Disease Neuroimaging I. 2013. Statistical analysis of longitudinal neuroimage data with Linear Mixed Effects models. Neuroimage. 66:249-260.

Bernal-Rusiel JL, Reuter M, Greve DN, Fischl B, Sabuncu MR, Alzheimer's Disease Neuroimaging I. 2013. Spatiotemporal linear mixed effects modeling for the mass-univariate analysis of longitudinal neuroimage data. Neuroimage. 81:358-370.

Carmona S, Zahs K, Wu E, Dakin K, Bras J, Guerreiro R. 2018. The role of TREM2 in Alzheimer's disease and other neurodegenerative disorders. Lancet Neurol. 17:721-730. 
Crary JF, Trojanowski JQ, Schneider JA, Abisambra JF, Abner EL, Alafuzoff I, Arnold SE, Attems J, Beach TG, Bigio EH, Cairns NJ, Dickson DW, Gearing M, Grinberg LT, Hof PR, Hyman BT, Jellinger K, Jicha GA, Kovacs GG, Knopman DS, Kofler J, Kukull WA, Mackenzie IR, Masliah E, McKee A, Montine TJ, Murray ME, Neltner JH, Santa-Maria I, Seeley WW, Serrano-Pozo A, Shelanski ML, Stein T, Takao M, Thal DR, Toledo JB, Troncoso JC, Vonsattel JP, White CL, 3rd, Wisniewski T, Woltjer RL, Yamada M, Nelson PT. 2014. Primary age-related tauopathy (PART): a common pathology associated with human aging. Acta neuropathologica. 128:755-766.

Dale AM, Fischl B, Sereno MI. 1999. Cortical surface-based analysis. I. Segmentation and surface reconstruction. Neuroimage. 9:179-194.

Desikan RS, Segonne F, Fischl B, Quinn BT, Dickerson BC, Blacker D, Buckner RL, Dale AM, Maguire RP, Hyman BT, Albert MS, Killiany RJ. 2006. An automated labeling system for subdividing the human cerebral cortex on MRI scans into gyral based regions of interest. Neuroimage. 31:968-980.

Fischl B, Salat DH, Busa E, Albert M, Dieterich M, Haselgrove C, van der Kouwe A, Killiany R, Kennedy D, Klaveness S, Montillo A, Makris N, Rosen B, Dale AM. 2002. Whole brain segmentation: automated labeling of neuroanatomical structures in the human brain. Neuron 33:341-355.

Fischl B, Sereno MI, Tootell RB, Dale AM. 1999. High-resolution intersubject averaging and a coordinate system for the cortical surface. Hum Brain Mapp. 8:272-284. 
Fjell AM, McEvoy L, Holland D, Dale AM, Walhovd KB. 2013. Brain changes in older adults at very low risk for Alzheimer's disease. The Journal of neuroscience : the official journal of the Society for Neuroscience. 33:8237-8242.

Fjell AM, McEvoy L, Holland D, Dale AM, Walhovd KB, Alzheimer's Disease Neuroimaging I. 2014. What is normal in normal aging? Effects of aging, amyloid and Alzheimer's disease on the cerebral cortex and the hippocampus. Prog Neurobiol. 117:20-40.

Folstein MF, Folstein SE, McHugh PR. 1975. "Mini-mental state". A practical method for grading the cognitive state of patients for the clinician. Journal of psychiatric research. 12:189-198.

Forabosco P, Ramasamy A, Trabzuni D, Walker R, Smith C, Bras J, Levine AP, Hardy J, Pocock JM, Guerreiro R, Weale ME, Ryten M. 2013. Insights into TREM2 biology by network analysis of human brain gene expression data. Neurobiology of aging. 34:2699-2714.

French L, Paus T. 2015. A FreeSurfer view of the cortical transcriptome generated from the Allen Human Brain Atlas. Front Neurosci. 9:323.

Gispert JD, Suarez-Calvet M, Monte GC, Tucholka A, Falcon C, Rojas S, Rami L, Sanchez-Valle R, Llado A, Kleinberger G, Haass C, Molinuevo JL. 2016. Cerebrospinal fluid STREM2 levels are associated with gray matter volume increases and reduced diffusivity in early Alzheimer's disease. Alzheimer's \& dementia : the journal of the Alzheimer's Association. 12:1259-1272. 
Glodzik L, Mosconi L, Tsui W, de Santi S, Zinkowski R, Pirraglia E, Rich KE, McHugh P, Li Y, Williams S, Ali F, Zetterberg H, Blennow K, Mehta P, de Leon MJ. 2012. Alzheimer's disease markers, hypertension, and gray matter damage in normal elderly. Neurobiology of aging. 33:1215-1227.

Gorbach T, Pudas S, Lundquist A, Oradd G, Josefsson M, Salami A, de Luna X, Nyberg L. 2017. Longitudinal association between hippocampus atrophy and episodic-memory decline. Neurobiology of aging. 51:167-176.

Guerreiro R, Wojtas A, Bras J, Carrasquillo M, Rogaeva E, Majounie E, Cruchaga C, Sassi C, Kauwe JS, Younkin S, Hazrati L, Collinge J, Pocock J, Lashley T, Williams J, Lambert JC, Amouyel P, Goate A, Rademakers R, Morgan K, Powell J, St George-Hyslop P, Singleton A, Hardy J. 2013. TREM2 variants in Alzheimer's disease. The New England journal of medicine. 368:117-127.

Hansson O, Zetterberg H, Buchhave P, Londos E, Blennow K, Minthon L. 2006. Association between CSF biomarkers and incipient Alzheimer's disease in patients with mild cognitive impairment: a follow-up study. The Lancet Neurology. 5:228-234.

Hawrylycz MJ, Lein ES, Guillozet-Bongaarts AL, Shen EH, Ng L, Miller JA, van de Lagemaat LN, Smith KA, Ebbert A, Riley ZL, Abajian C, Beckmann CF, Bernard A, Bertagnolli D, Boe AF, Cartagena PM, Chakravarty MM, Chapin M, Chong J, Dalley RA, David Daly B, Dang C, Datta S, Dee N, Dolbeare TA, Faber V, Feng D, Fowler DR, Goldy J, Gregor BW, Haradon Z, Haynor DR, Hohmann JG, Horvath S, Howard RE, Jeromin A, Jochim JM, Kinnunen M, Lau C, Lazarz ET, Lee C, Lemon TA, Li L, Li Y, Morris JA, Overly CC, Parker PD, Parry SE, Reding M, Royall JJ, Schulkin J, Sequeira PA, Slaughterbeck CR, Smith SC, Sodt AJ, Sunkin SM, Swanson BE, Vawter MP, Williams D, Wohnoutka P, Zielke HR, 
Geschwind DH, Hof PR, Smith SM, Koch C, Grant SGN, Jones AR. 2012. An anatomically comprehensive atlas of the adult human brain transcriptome. Nature. 489:391-399.

Heneka MT, Carson MJ, El Khoury J, Landreth GE, Brosseron F, Feinstein DL, Jacobs AH, Wyss-Coray T, Vitorica J, Ransohoff RM, Herrup K, Frautschy SA, Finsen B, Brown GC, Verkhratsky A, Yamanaka K, Koistinaho J, Latz E, Halle A, Petzold GC, Town T, Morgan D, Shinohara ML, Perry VH, Holmes C, Bazan NG, Brooks DJ, Hunot S, Joseph B, Deigendesch N, Garaschuk O, Boddeke E, Dinarello CA, Breitner JC, Cole GM, Golenbock DT, Kummer MP. 2015. Neuroinflammation in Alzheimer's disease. The Lancet Neurology. 14:388-405.

Henjum K, Almdahl IS, Arskog V, Minthon L, Hansson O, Fladby T, Nilsson LN. 2016. Cerebrospinal fluid soluble TREM2 in aging and Alzheimer's disease. Alzheimer's research \& therapy. 8:17.

Heslegrave A, Heywood W, Paterson R, Magdalinou N, Svensson J, Johansson P, Ohrfelt A, Blennow K, Hardy J, Schott J, Mills K, Zetterberg H. 2016. Increased cerebrospinal fluid soluble TREM2 concentration in Alzheimer's disease. Molecular neurodegeneration. 11:3.

Hickman S, Izzy S, Sen P, Morsett L, El Khoury J. 2018. Microglia in neurodegeneration. Nat Neurosci. 21:1359-1369.

Idland AV, Sala-Llonch R, Borza T, Watne LO, Wyller TB, Braekhus A, Zetterberg H, Blennow K, Walhovd KB, Fjell AM. 2016. CSF neurofilament light levels predict hippocampal atrophy in cognitively healthy older adults. Neurobiology of aging. 49:138-144. 
Jack CR, Jr., Bennett DA, Blennow K, Carrillo MC, Dunn B, Haeberlein SB, Holtzman DM, Jagust W, Jessen F, Karlawish J, Liu E, Molinuevo JL, Montine T, Phelps C, Rankin KP, Rowe CC, Scheltens P, Siemers E, Snyder HM, Sperling R. 2018. NIA-AA Research Framework: Toward a biological definition of Alzheimer's disease. Alzheimer's \& dementia : the journal of the Alzheimer's Association. 14:535562.

Jack CR, Jr., Bennett DA, Blennow K, Carrillo MC, Feldman HH, Frisoni GB, Hampel H, Jagust WJ, Johnson KA, Knopman DS, Petersen RC, Scheltens P, Sperling RA, Dubois B. 2016. A/T/N: An unbiased descriptive classification scheme for Alzheimer disease biomarkers. Neurology. 87:539-547.

Jack CR, Jr., Knopman DS, Chetelat G, Dickson D, Fagan AM, Frisoni GB, Jagust W, Mormino EC, Petersen RC, Sperling RA, van der Flier WM, Villemagne VL, Visser PJ, Vos SJ. 2016. Suspected nonAlzheimer disease pathophysiology--concept and controversy. Nature reviews Neurology. 12:117124.

Jack CR, Jr., Knopman DS, Jagust WJ, Shaw LM, Aisen PS, Weiner MW, Petersen RC, Trojanowski JQ. 2010. Hypothetical model of dynamic biomarkers of the Alzheimer's pathological cascade. The Lancet Neurology. 9:119-128.

Jay TR, Miller CM, Cheng PJ, Graham LC, Bemiller S, Broihier ML, Xu G, Margevicius D, Karlo JC, Sousa GL, Cotleur AC, Butovsky O, Bekris L, Staugaitis SM, Leverenz JB, Pimplikar SW, Landreth GE, Howell GR, Ransohoff RM, Lamb BT. 2015. TREM2 deficiency eliminates TREM2+ inflammatory macrophages and ameliorates pathology in Alzheimer's disease mouse models. The Journal of experimental medicine. 212:287-295. 
Jay TR, von Saucken VE, Landreth GE. 2017. TREM2 in Neurodegenerative Diseases. Molecular neurodegeneration. 12:56.

Jiang T, Tan L, Zhu XC, Zhou JS, Cao L, Tan MS, Wang HF, Chen Q, Zhang YD, Yu JT. 2015. Silencing of TREM2 exacerbates tau pathology, neurodegenerative changes, and spatial learning deficits in P301S tau transgenic mice. Neurobiology of aging. 36:3176-3186.

Jovicich J, Marizzoni M, Sala-Llonch R, Bosch B, Bartres-Faz D, Arnold J, Benninghoff J, Wiltfang J, Roccatagliata L, Nobili F, Hensch T, Trankner A, Schonknecht P, Leroy M, Lopes R, Bordet R, Chanoine V, Ranjeva JP, Didic M, Gros-Dagnac H, Payoux P, Zoccatelli G, Alessandrini F, Beltramello A, Bargallo N, Blin O, Frisoni GB. 2013. Brain morphometry reproducibility in multi-center 3T MRI studies: a comparison of cross-sectional and longitudinal segmentations. Neuroimage. 83:472-484.

Kendrick DC, Gibson AJ, Moyes IC. 1979. The Revised Kendrick Battery: clinical studies. The British journal of social and clinical psychology. 18:329-340.

Kleinberger G, Yamanishi Y, Suarez-Calvet M, Czirr E, Lohmann E, Cuyvers E, Struyfs H, Pettkus N, Wenninger-Weinzierl A, Mazaheri F, Tahirovic S, Lleo A, Alcolea D, Fortea J, Willem M, Lammich S, Molinuevo JL, Sanchez-Valle R, Antonell A, Ramirez A, Heneka MT, Sleegers K, van der Zee J, Martin JJ, Engelborghs S, Demirtas-Tatlidede A, Zetterberg H, Van Broeckhoven C, Gurvit H, Wyss-Coray T, Hardy J, Colonna M, Haass C. 2014. TREM2 mutations implicated in neurodegeneration impair cell surface transport and phagocytosis. Science translational medicine. 6:243ra286. 
Krasemann S, Madore C, Cialic R, Baufeld C, Calcagno N, El Fatimy R, Beckers L, O'Loughlin E, Xu Y, Fanek Z, Greco DJ, Smith ST, Tweet G, Humulock Z, Zrzavy T, Conde-Sanroman P, Gacias M, Weng Z, Chen H, Tjon E, Mazaheri F, Hartmann K, Madi A, Ulrich JD, Glatzel M, Worthmann A, Heeren J, Budnik B, Lemere C, Ikezu T, Heppner FL, Litvak V, Holtzman DM, Lassmann H, Weiner HL, Ochando J, Haass C, Butovsky O. 2017. The TREM2-APOE Pathway Drives the Transcriptional Phenotype of Dysfunctional Microglia in Neurodegenerative Diseases. Immunity. 47:566-581.e569.

Leyns CEG, Ulrich JD, Finn MB, Stewart FR, Koscal L, Remolina Serrano J, Robinson GO, Anderson E, Colonna M, Holtzman DM. 2017. TREM2 deficiency attenuates neuroinflammation and protects against neurodegeneration in a mouse model of tauopathy. Proceedings of the National Academy of Sciences of the United States of America. 114:11524-11529.

McManus RM, Heneka MT. 2017. Role of neuroinflammation in neurodegeneration: new insights. Alzheimer's research \& therapy. 9:14.

Morris JC, Heyman A, Mohs RC, Hughes JP, van Belle G, Fillenbaum G, Mellits ED, Clark C. 1989. The Consortium to Establish a Registry for Alzheimer's Disease (CERAD). Part I. Clinical and neuropsychological assessment of Alzheimer's disease. Neurology. 39:1159-1165.

Nyberg L, Lovden M, Riklund K, Lindenberger U, Backman L. 2012. Memory aging and brain maintenance. Trends Cogn Sci. 16:292-305. 
Pudas S, Persson J, Josefsson M, de Luna X, Nilsson LG, Nyberg L. 2013. Brain characteristics of individuals resisting age-related cognitive decline over two decades. The Journal of neuroscience : the official journal of the Society for Neuroscience. 33:8668-8677.

Ransohoff RM. 2016. How neuroinflammation contributes to neurodegeneration. Science (New York, NY). 353:777-783.

Rauchmann BS, Schneider-Axmann T, Alexopoulos P, Perneczky R. 2018. CSF soluble TREM2 as a measure of immune response along the Alzheimer's disease continuum. Neurobiology of aging. 74:182-190.

Regen F, Hellmann-Regen J, Costantini E, Reale M. 2017. Neuroinflammation and Alzheimer's Disease: Implications for Microglial Activation. Curr Alzheimer Res. 14:1140-1148.

Reitan RM. 1955. The relation of the trail making test to organic brain damage. Journal of consulting psychology. 19:393-394.

Reuter M, Schmansky NJ, Rosas HD, Fischl B. 2012. Within-subject template estimation for unbiased longitudinal image analysis. Neuroimage. 61:1402-1418.

Shin J, French L, Xu T, Leonard G, Perron M, Pike GB, Richer L, Veillette S, Pausova Z, Paus T. 2018. Cell-Specific Gene-Expression Profiles and Cortical Thickness in the Human Brain. Cerebral cortex (New York, NY : 1991). 28:3267-3277. 
Spreen O, Strauss, E., Sherman, E.M.S 1991. A Compendium of Neuropsychological Tests:

Administration, Norms, and Commentary. New York: Oxford University Press

Streit WJ, Braak H, Xue QS, Bechmann I. 2009. Dystrophic (senescent) rather than activated microglial cells are associated with tau pathology and likely precede neurodegeneration in Alzheimer's disease. Acta neuropathologica. 118:475-485.

Suarez-Calvet M, Araque Caballero MA, Kleinberger G, Bateman RJ, Fagan AM, Morris JC, Levin J, Danek A, Ewers M, Haass C. 2016. Early changes in CSF sTREM2 in dominantly inherited Alzheimer's disease occur after amyloid deposition and neuronal injury. Science translational medicine. 8:369ra178.

Suarez-Calvet M, Kleinberger G, Araque Caballero MA, Brendel M, Rominger A, Alcolea D, Fortea J, Lleo A, Blesa R, Gispert JD, Sanchez-Valle R, Antonell A, Rami L, Molinuevo JL, Brosseron F, Traschutz A, Heneka MT, Struyfs H, Engelborghs S, Sleegers K, Van Broeckhoven C, Zetterberg H, Nellgard B, Blennow K, Crispin A, Ewers M, Haass C. 2016. sTREM2 cerebrospinal fluid levels are a potential biomarker for microglia activity in early-stage Alzheimer's disease and associate with neuronal injury markers. EMBO molecular medicine. 8:466-476.

Suarez-Calvet M, Morenas-Rodriguez E, Kleinberger G, Schlepckow K, Araque Caballero MA, Franzmeier N, Capell A, Fellerer K, Nuscher B, Eren E, Levin J, Deming Y, Piccio L, Karch CM, Cruchaga C, Shaw LM, Trojanowski JQ, Weiner M, Ewers M, Haass C. 2019. Early increase of CSF sTREM2 in Alzheimer's disease is associated with tau related-neurodegeneration but not with amyloid-beta pathology. Molecular neurodegeneration. 14:1. 
Wood SN. 2006. Generalized Additive Models: An Introduction with R: Chapman and Hall/CRC.

Wyss-Coray T. 2016. Ageing, neurodegeneration and brain rejuvenation. Nature. 539:180-186.

Zetterberg H. 2017. Review: Tau in biofluids - relation to pathology, imaging and clinical features. Neuropathology and applied neurobiology. 43:194-199. 


\section{TABLES}

Table 1 Population characteristics at baseline. Participants were classified according to the A (amyloid deposition) /T (aggregated tau)/(N)-framework by applying CSF measures with the following cut-off values: $\mathrm{A}+<\mathrm{A} \beta 42530 \mathrm{pg} / \mathrm{ml}<\mathrm{A}-$ and $\mathrm{T}+>$ p-tau $60 \mathrm{pg} / \mathrm{ml}>\mathrm{T}$. Except for sex, values are median (inter-quartile range). Age and education are given in years. CSF concentrations are given in pg/ml, except CSF-sTREM2 in $\mathrm{ng} / \mathrm{ml}$.

\begin{tabular}{|c|c|c|c|c|c|}
\hline & All $(n=115)$ & A-T- $(n=50)$ & $A-T+(n=33)$ & $A+T+(n=15)$ & $A+T-(n=15)$ \\
\hline Age & 71 (68-76) & 71 (67-75) & 73 (70-76) & 74 (69-79) & 71 (67-77) \\
\hline Female, n (\%) & $57(53)$ & $22(47)$ & $18(60)$ & $9(64)$ & $6(43)$ \\
\hline Education $^{a}$ & $14(12-17)$ & $16(13-18)$ & $14(11-17)$ & $13(11-16)$ & $12(11-16)$ \\
\hline CSF-sTREM2 & $8(6-11)$ & $7(6-10)$ & $10(8-14)$ & $10(8-11)$ & $6(5-8)$ \\
\hline CSF-t-tau ${ }^{b}$ & $324(268-485)$ & $282(225-329)$ & $485(400-553)$ & $507(488-766)$ & $272(187-322)$ \\
\hline CSF-p-tau ${ }^{b}$ & $58(45-74)$ & $48(43-54)$ & $74(65-89)$ & $81(74-101)$ & $42(33-57)$ \\
\hline CSF- $A \beta-42^{b}$ & $727(513-852)$ & $793(660-859)$ & 851 (710- 979) & $469(423-490)$ & $471(380-502)$ \\
\hline Left hippo & $3505(412)$ & 3587 (415) & 3459 (367) & 3341 (555) & 3528 (309) \\
\hline Right hippo & 3541 (406) & $3628(373)$ & 3465 (414) & 3419 (528) & 3601 (295) \\
\hline
\end{tabular}

Abbreviation: $\mathrm{p}$-tau: phosphorylated tau; t-tau: total tau.

a 3 missing values; ${ }^{b} 2$ missing values. Left/right hippo: Hippocampal volume at baseline. 
Table 2 Virtual histology results.

\begin{tabular}{lccll} 
Cell Type & nGenes & average & $\mathbf{p}$ & FDR-p \\
\hline Astrocyte & 54 & 0.064 & 0.079 & 0.18 \\
CA1.Pyramidal & 103 & 0.161 & $<\mathbf{0 . 0 0 1}$ & $<\mathbf{0 . 0 0 1}$ \\
Endothelial & 57 & -0.07 & 0.05 & 0.16 \\
Ependymal & 84 & 0.034 & 0.258 & 0.33 \\
Interneuron & 100 & 0.035 & 0.20 & 0.33 \\
Microglia & 48 & 0.105 & 0.007 & 0.03 \\
Mural & 25 & 0.025 & 0.65 & 0.73 \\
Oligodendrocyte & 60 & -0.04 & 0.26 & 0.33 \\
S1. Pyramidal & 73 & 0.011 & 0.73 & 0.73
\end{tabular}

Virtual histology results. nGenes $=$ number of genes in each cell type panel; average $=$ mean average of the TREM2-related cortical thinning - gene expression inter-regional correlation; $p$-values and False discovery rate (FDR)-corrected $p$-values with respect to the empirical null distribution. Bold $p<$ 0.001, Italics $p<0.05$ 


\section{CAPTIONS TO FIGURES}

\section{Figure 1 Relationship between sTREM2 and cortical thinning}

Panel A: Relationship between baseline levels of sTREM2 on cortical thinning. Panel B:

Same analysis as in A, with levels of A $\beta 42$ regressed out. Panel C: A $\beta 42$ and total-tau regressed out. In all panels, the clusters survived corrections for multiple comparisons.

\section{Figure 2 Interactions between sTREM2 and time}

Panel A: Interactions between sTREM2 and cortical thickness over time in the three significant clusters. The contour plots illustrate how cortical thickness varies as a function of interval since baseline (time) and sTREM2 levels. As can be seen, blue colors, denoting thinner cortex, are seen for participants with high levels of sTREM2 over time. Panel B and C: Spaghetti plots illustrating the relationship between thickness change as a function of time in participants with normal $(\mathrm{z}<1$, red markers, Panel B) or high $(\mathrm{z} \geq 1$, blue markers, Panel C) levels of sTREM2. The shaded region around the fit lines denotes +/-2 SE.

\section{Figure 3 Relationship between sTREM2 and hippocampal atrophy}

Panel A: Contour and spaghetti plot illustrating how left hippocampal volume varies as a function of interval since baseline and sTREM2 levels. Panel B: Right hippocampal volume.

\section{Figure 4 Relationships between sTREM2 and memory change}

Contour (Panel A) and spaghetti plots (Panel B) illustrating the relationship between interval since baseline and change in memory score as a function of baseline sTREM2 levels. The shaded region around the fit lines denotes $+/-2 \mathrm{SE}$.

\section{Figure 5 Virtual histology}


Each plot corresponds to one of the 9 different cell type panels and shows the distribution of the expression-sTREM2-related thinning correlation coefficients for cell-specific genes in each cell-type group as a density function and as a cloud of dots. The x-axis represents the Pearson's correlation between sTREM2-related thinning and expression profiles for a given set of cell-specific marker genes while the y-axis indicates the probability density for the correlations across the cell-specific genes. The vertical black line indicates the average expression-thinning correlation coefficient across all genes within a cell-type group while the shaded gray box indicates the $95 \%$ limits of the empirical null distribution, thus indicating $5 \%$ significance level unadjusted between cell-type comparisons. 